\title{
The incidence game chromatic number
}

\author{
Stephan Dominique Andres \\ Zentrum für angewandte Informatik Köln, \\ University of Cologne, \\ Weyertal 80, 50931 Köln, Germany
}

\begin{abstract}
We introduce the incidence game chromatic number which unifies the ideas of game chromatic number and incidence coloring number of an undirected graph. For $k$ degenerate graphs with maximum degree $\Delta$, the upper bound $2 \Delta+4 k-2$ for the incidence game chromatic number is given. If $\Delta \geq 5 k$, we improve this bound to the value $2 \Delta+3 k-1$. We also determine the exact incidence game chromatic number of cycles, stars and sufficiently large wheels and obtain the lower bound $\frac{3}{2} \Delta$ for the incidence game chromatic number of graphs of maximum degree $\Delta$.
\end{abstract}

Key words: incidence coloring number, game chromatic number, $k$-degenerate graph, star, wheel, cycle, game chromatic index

2000 MSC: 05C15, 91A05

\section{Introduction}

Let $G=(V, E)$ be a graph with vertex set $V$ and edge set $E$. The set of incidences of $G$ is defined as

$$
I=\{(v, e) \in V \times E \mid v \text { is incident with } e\} .
$$

Two distinct incidences $(v, e),(w, f) \in I$ are adjacent if $(v, f) \in I$ or $(w, e) \in I$. This means in particular, if either $v=w$ or $e=f$, then the incidences $(v, e)$ and $(w, f)$ are adjacent. See Fig. 1.

Email address: andres@zpr.uni-koeln.de (Stephan Dominique Andres).

URL: http://www.math.uni-koeln.de/ andres (Stephan Dominique Andres).

Preprint submitted to Discrete Applied Mathematics 28 August 2007 


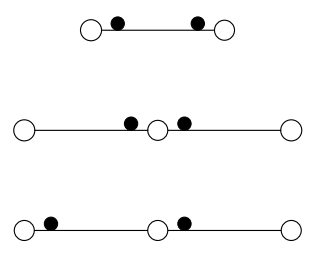

(a)

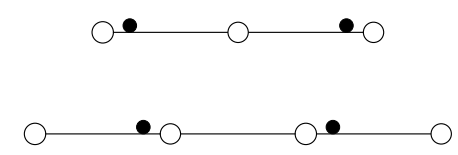

(b)

Fig. 1. (a) pairs of adjacent incidences (b) pairs of non-adjacent incidences. A white dot denotes a vertex, a black dot on an edge $e$ next to a vertex $v$ denotes the incidence $(v, e)$.

Consider the following game which is played on $I$ with a color set $C$. Two players, Alice and Bob, alternately color an incidence with a color from $C$ in such a way that incidences that are adjacent receive distinct colors. The game ends when this is not possible any more. Alice wins if every incidence is colored at the end of the game, otherwise Bob wins. The smallest number of colors, so that Alice has a winning strategy for the game played on $I$, is called incidence game chromatic number $\iota_{g}(G)$ of $G$. This is a competitive version of the incidence coloring number introduced by Brualdi and Massey [3].

The game chromatic number $\chi_{g}(G)$ (resp. game chromatic index $\chi_{g}^{\prime}(G)$ ) of a graph $G$ is defined for a similar game where vertices (resp. edges) of a graph are colored instead of incidences, and where in the definition of the game the adjacency of incidences is replaced by the adjacency of vertices (resp. edges). It was introduced by Bodlaender [2] (resp. Cai and Zhu [4]). To be precise, we assume in our games that Alice has the first move, and missing a turn is not allowed.

If $G$ is a graph, then $G^{I}$ is the graph the vertices of which are the incidences of $G$, with edges joining adjacent incidences. Thus $\iota_{g}(G)=\chi_{g}\left(G^{I}\right)$. However, in this paper we will only make use of this construction in the proof of Theorem 3 .

An incidence coloring of a graph $G$ is a color assignment to its incidences in such a way that adjacent incidences receive distinct colors. The minimum number of colors needed for $G$ is the incidence coloring number of $G$. An incidence of an edge $e=v w$ is one of the incidences $(v, e)$ or $(w, e)$. Later we will also consider the incidences of an oriented graph. In this case, in all previous definitions the edges have to be replaced by arcs.

Upper bounds for the incidence coloring number have been determined for several classes of graphs, e.g. for $k$-degenerate graphs, $K_{4}$-minor free graphs and planar graphs [9], and graphs with maximum degree 3 [13]. These bounds depend on the maximum degree of the graphs. Guiduli [8] found the tight asymptotic upper bound $\Delta+O(\log \Delta)$ for the incidence coloring number of graphs with maximum degree $\Delta$. For forests [3], Halin graphs of maximum degree $\Delta \geq 5$ and outerplanar graphs of maximum degree $\Delta \geq 4$ [14], certain 
types of meshes [10], and complete $k$-partite graphs [12] the exact values of the incidence coloring numbers are known.

Upper bounds for the game chromatic number have been determined for forests [6], outerplanar graphs [7], graphs embeddable in an orientable surface [11], line graphs of $k$-degenerate graphs [4], line graphs of forests with maximum degree $\Delta \neq 4[4,5,1]$, and planar graphs $[11,15]$.

When coloring incidences one should be aware of the unusal properties of the adjacency relation of incidences. As depicted in Fig. 2, both incidences of the edges incident with $v$ (except $v w$ ) are adjacent to the incidence $(v, v w)$, whereas only one incidence of the edges incident with $w$ is adjacent to the incidence $(v, v w)$, and the incidences of other edges (which are not depicted) are not adjacent to $(v, v w)$. Since the degree of $v$ is $m=4$ and the degree of $w$ is $n=6$, the number of incidences adjacent to $(v, v w)$ is $2(m-1)+1+(n-1)=12$ in our example. Therefore $(v, v w)$ can be colored with a 13-th color.

A trivial upper bound for the incidence game chromatic number of graphs $G_{\Delta}$ with maximum degree $\Delta \geq 1$ is

$$
\iota_{g}\left(G_{\Delta}\right) \leq 3 \Delta-1
$$

This is simply the maximum number of adjacent incidences an incidence can have plus one. Our main theorem improves this bound for $k$-degenerate graphs (Section 2.) The trivial upper bound is attained by sufficiently large cycles, as we shall see in Section 3. In Section 4 we determine the exact incidence game chromatic number of stars and wheels. When we use the ideas of these proofs we obtain a lower bound for the incidence game chromatic number of graphs with maximum degree $\Delta$, a bound which is half of the trivial upper bound. This phenomenon also occurs for the game chromatic index, the trivial lower bound of which is half of its trivial upper bound.

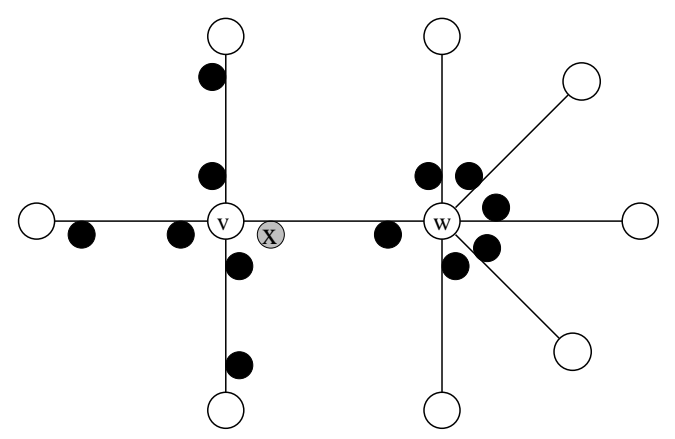

Fig. 2. The incidences adjacent to the incidence $x=(v, v w)$ are indicated by black dots and the incidence $(v, v w)$ is depicted by a grey dot with ' $\mathrm{x}$ '. Here a black dot on an edge $f$ next to a vertex $z$ means the incidence $(z, f)$. 


\section{$2 k$-degenerate graphs}

In this section we will consider the incidence game chromatic number of $k$ degenerate graphs. The proof uses an activation strategy. The idea of such an activation strategy was already used by Cai and Zhu [4] in order to bound the game chromatic index of $k$-degenerate graphs.

We start with a definition. A graph $G$ with vertex set $\left\{v_{1}, v_{2}, \ldots, v_{n}\right\}$ is $k$ degenerate if there is a linear order

$$
L\left(v_{1}\right)<L\left(v_{2}\right)<\cdots<L\left(v_{n}\right)
$$

on its vertex set such that for every $1 \leq i \leq n$ the vertex $v_{i}$ has degree at most $k$ in the induced subgraph on the vertex set $\left\{v_{1}, v_{2}, \ldots, v_{i}\right\}$. We will prove the following

Theorem 1 For a $k$-degenerate graph $G$ with maximum degree $\Delta$ we have

(a) $\iota_{g}(G) \leq 2 \Delta+4 k-2$.

(b) $\iota_{g}(G) \leq 2 \Delta+3 k-1$, provided $\Delta \geq 5 k-1$.

(c) $\iota_{g}(G) \leq \Delta+8 k-2$, provided $\Delta \leq 5 k-1$.

PROOF. We will describe a winning strategy of Alice with $\max \{\Delta+8 k-2$, $2 \Delta+3 k-1\}$ colors. Before doing so we need some preparations. The vertex set $\left\{v_{1}, v_{2}, \ldots, v_{n}\right\}$ of $G$ is considered as ordered according to a linear order $L$, i.e.

$$
L\left(v_{1}\right)<L\left(v_{2}\right)<\cdots<L\left(v_{n}\right)
$$

so that, for every $1 \leq i \leq n, v_{i}$ has vertex degree at most $k$ in the induced subgraph on $\left\{v_{1}, v_{2}, \ldots, v_{i}\right\}$. Such an order exists, since $G$ is $k$-degenerate. To every edge $v_{i} v_{j}$ is assigned an orientation in such a way that if $L\left(v_{i}\right)>L\left(v_{j}\right)$ then $\left(v_{i}, v_{j}\right)$ is an arc (directed edge). We call the digraph created this way $\vec{G}$. Every time we color an incidence, we color it in both $G$ and $\vec{G}$. Let $d^{+}(v)$ be the number of out-arcs $(v, w)$ of $v$. By construction, $d^{+}(v) \leq k$ for every vertex $v$. A sink is a vertex $v$ with $d^{+}(v)=0$. The level of vertex $v_{i}$ is the number $i$. The level of an arc is the level of its starting vertex.

During the game certain arcs are considered as active, all other arcs as inactive. A subset of the active arcs is represented by the half-selected resp. selected arcs, i.e. those arcs one incidence or both incidences of which are colored. By an arc of minimum level we mean an uncolored or half-selected arc the level of which is minimal among all such arcs. For an $\operatorname{arc}(v, w)$, we call the 
incidence $(v,(v, w))$ top incidence of $(v, w)$, and the incidence $(w,(v, w))$ is called bottom incidence of $(v, w)$. An arc is called top-half-selected if its top incidence is colored and its bottom incidence is uncolored.

At the beginning Alice colors a bottom incidence of an arc incident with a sink and activates its arc. After that, for each of Bob's moves, where Bob colors an incidence of an $\operatorname{arc}(v, w)$, she answers as follows:

(Step 0) $\quad e=(v, w)$, activate $e$

(Step 1) while $w$ has inactive out-arcs do

choose an inactive out-arc $\left(w, w_{1}\right)$, activate it,

$w:=w_{1}$

end do

(Step 2) if $w$ has an unselected or top-half-selected active out-arc $e$,

(Step 2a) color the bottom incidence of $e$,

else if $w$ has an half-selected active out-arc $e$,

(Step 2b) color the uncolored incidence of $e$,

(Step 2c) else color an incidence of an $\operatorname{arc} e$ of minimum level, activate $e$ (in Step 2c color a bottom incidence if possible)

We will show that for this strategy after every move of Alice every uncolored incidence of an unselected or half-selected $\operatorname{arc}(v, w)$ has at $\operatorname{most} \max \{2 \Delta+$ $3 k-3, \Delta+8 k-4\}$ colored adjacent incidences. Note that, in Step 2c, no arc $(x, v)$ can be (half-)selected before $(v, w)$ by Alice as $(v, w)$ lies on a lower level. Therefore $\operatorname{arcs}(x, v)$ can only pass through steps $1,2 \mathrm{a}$, and $2 \mathrm{~b}$ before both incidences of $(v, w)$ are colored. There are at most $\# v_{\text {out }}=k-1$ arcs $(v, y)$ different from $(v, w)$. So at most $k$ arcs $(x, v)$ can have been activated (Step 1) in order to activate arcs of the type $(v, y)$. After that at most $2 k-1$ $\operatorname{arcs}(x, v)$ can have been activated (Steps 1, 2a, 2b) in order to color $2 k-1$

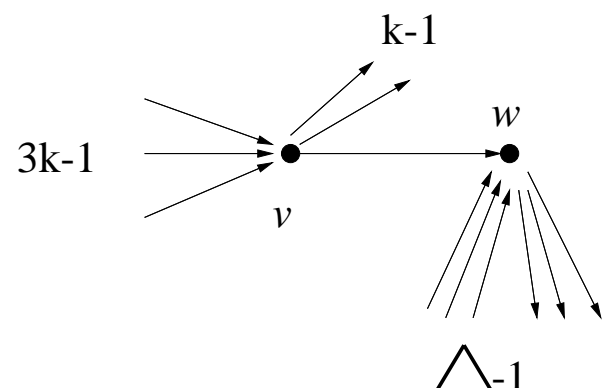

Fig. 3. Adjacent incidences of arc $(v, w)$ which may be colored 
of the $2 k$ incidences of the $\operatorname{arcs}(v, y)$. Thus there are at most $\# v_{i n}=3 k-1$ active arcs of the form $(x, v)$. In the worst case there are $\# w=\Delta-1$ active arcs incident with $w$. See Fig. 3. So, in the worst case the incidence $(v,(v, w))$ has at most

$$
\# w+1+2 \# v_{\text {out }}+2 \# v_{\text {in }}=\Delta+8 k-4
$$

adjacent incidences on active arcs, and the incidence $(w,(v, w))$ has at most

$$
2 \# w+1+\# v_{\text {out }}+\# v_{\text {in }}=2 \Delta+4 k-3
$$

adjacent incidences on active arcs after a move of Alice.

However, we need not count the number of adjacent incidences on active arcs, but only the number of colored adjacent incidences. The latter number is smaller than the previous since the set of selected and half-selected arcs is a subset of the set of active arcs, and since Alice, by her strategy, prefers to color bottom incidences. Therefore, when the incidence $(w,(v, w))$ is to be colored, the top incidence of any arc of type $(v, y)$ is either uncolored or colored by Bob. If $\# v_{\text {out }}^{\prime}=m$ top incidences of the arcs of type $(v, y)$ (including $(v, w)$ ) are colored by Bob, then, by the same reasons as before, there are at most $\# v_{i n}^{\prime}=3 k-1-m$ active arcs of the form $(x, v)$. Thus the incidence $(w,(v, w))$ has at most

$$
2 \# w+\# v_{\text {out }}^{\prime}+\# v_{\text {in }}^{\prime}=2 \Delta+3 k-3
$$

colored adjacent incidences.

Summarizing, we state that after Alice's move an incidence can have at most

$$
\max \{\Delta+8 k-4,2 \Delta+3 k-3\}
$$

colored adjacent incidences. After Bob's next move an incidence can have at most

$$
\max \{\Delta+8 k-3,2 \Delta+3 k-2\}
$$

colored adjacent incidences. We obtain the conclusion that

$$
\max \{\Delta+8 k-2,2 \Delta+3 k-1\}
$$

colors are sufficient to color every incidence during the game. 
Further we have $\Delta+8 k-2 \leq 2 \Delta+3 k-1$ if, and only if, $\Delta \geq 5 k-1$, from which (b) and (c) follows. Note that, for $\Delta \geq 4 k, \Delta+8 k-2 \leq 2 \Delta+4 k-2$, and for $\Delta \leq 4 k-1$, the trivial upper bound $3 \Delta-1$ is better than the bound $b_{0}=2 \Delta+4 k-2$. Thus $b_{0}$ is an upper bound for all $\Delta$, as stated in (a).

The game chromatic index of a $k$-degenerate graph of maximum degree $\Delta$ is at most $\Delta+3 k-1[4]$. Our bound is better than simply doubling this bound.

Corollary 2 Let $P$ be a planar graph with maximum degree $\Delta, O$ be an outerplanar graph with maximum degree $\Delta$, and $F$ be a forest with maximum degree $\Delta$. Then

$$
\iota_{g}(P) \leq 2 \Delta+18, \quad \iota_{g}(O) \leq 2 \Delta+6, \quad \iota_{g}(F) \leq 2 \Delta+2
$$

PROOF. This follows from the fact that planar graphs are 5-degenerate, outerplanar graphs are 2-degenerate, and forests are 1-degenerate.

The results of this section have a noncompetitive analogue: Hosseini Dolama et al. [9] found $\Delta+2 k-1$ as an upper bound for the incidence coloring number of $k$-degenerate graphs and $\Delta+7$ as an upper bound for the incidence coloring number of planar graphs of maximum degree $\Delta$.

\section{Cycles}

In this section we consider the incidence game chromatic number of cycles. While the incidence game chromatic number of cycles attains the trivial upper bound (1), its counterparts for stars and wheels, in Section 4, will make us discover a trivial lower bound that cannot be improved for graphs in general. Let $C_{k}$ be the cycle with $k$ vertices.

Theorem $3 \quad \iota_{g}\left(C_{k}\right)=5$ for $k \geq 7$.

PROOF. Let $C_{k}$ be a cycle with $k \geq 7$ edges. Then $C_{k}^{I}=\left(V^{I}, E^{I}\right)$ is the graph with vertex set $V^{I}=\left\{v_{1}, v_{2}, \ldots, v_{2 k}\right\}$ and edge set

$$
E^{I}=\left\{v_{i} v_{j} \mid i-j \equiv \pm 1, \pm 2 \bmod 2 k\right\}
$$

$\iota_{g}\left(C_{k}\right) \leq 5$ follows from the trivial upper bound. So, we only have to prove $\iota_{g}\left(C_{k}\right)=\chi_{g}\left(C_{k}^{I}\right) \geq 5$, i.e. we have to explain a winning strategy for Bob for 


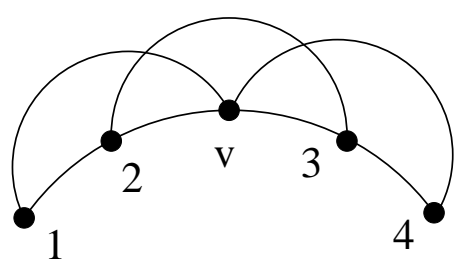

Fig. 4. A winning configuration for Bob on a cycle. If there are only 4 colors in the game, the uncolored vertex $v$ cannot be colored any more.

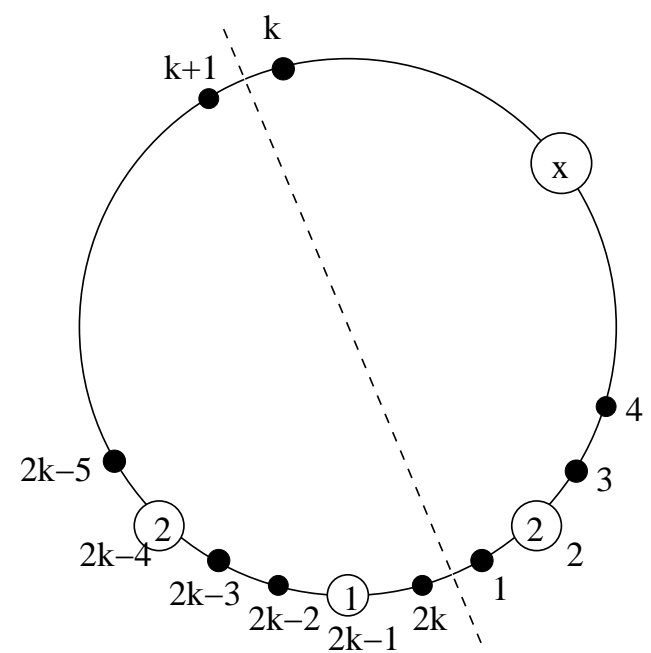

Fig. 5. State of the game after Bob's second move. Some vertices of of $C_{k}^{I}$ are black dots, colored vertices are white dots with the number of the color in it.

the vertex coloring game with 4 or less colors on $C_{k}^{I}$. It is easy to see that Bob wins with 3 or less colors. Assume for the following that the players have 4 colors at their disposal. The general winning idea of Bob is to construct a configuration as in Fig. 4.

W.l.o.g. Alice starts the game by coloring $v_{2 k-1}$ with color 1 . Bob then colors a vertex at distance 3, namely $v_{2}$ with color 2 . By reasons of symmetry we may assume that Alice, in her next move, colors one of the vertices $v_{3}, \ldots, v_{k}$, and if she colors $v_{3}$, then she uses color 1 . Indeed if Alice colored $v_{2 k}$ or $v_{1}$ (with color 3 ), Bob would color $v_{3}$ or $v_{2 k-2}$ at distance 3 from Alice's vertex with color 4 , so that $v_{1}$ or $v_{2 k}$ could not be colored any more. In the same way, if Alice colored $v_{3}$ with color 3 , Bob would color $v_{2 k}$ with color 4 , which would result in a win for him since he has constructed a winning configuration around the uncolored vertex $v_{1}$. Since the vertex Alice has colored in her second move is far enough away from $v_{2 k-4}$, Bob can color $v_{2 k-4}$ with color 2 in his second move. (Here we need the precondition $k \geq 7$, from which $(2 k-4)-k \geq 3$ follows.) The situation of the game after Bob's second move is depicted in Fig. 5.

Now, Alice is stuck. By the same reason as discussed above, Alice may not color $v_{2 k-5}$ or $v_{3}$ with a color different from 1 . Furthermore, if she colors 
$v_{2 k-3}, v_{2 k-2}, v_{2 k}$ or $v_{1}$, Bob can force a win in his next move. The next moves until Bob has created a winning configuration or until there are only 5 uncolored vertices left will be called a round. Everything Alice can do is to color the remaining of the vertices $v_{3}, \ldots, v_{2 k-5}$ in the round. Bob will color only vertices $v_{4}, \ldots, v_{2 k-6}$ in the round. Either Bob wins or the round ends when there are only 5 uncolored vertices left. Consider the latter case.

If Alice, in her last move, has colored $v_{3}$ with a color different from color 1 , Bob can force a win as described above. Thus we may assume without loss of generality that $v_{3}$ is colored with color 1 . We distinguish two cases.

The first case is that one of the vertices $v_{4}, \ldots, v_{2 k-6}$ is uncolored or $v_{2 k-5}$ is uncolored but can be colored with color 1. Then Bob colors this vertex, preferably with color 1 . Now the only uncolored vertices are $v_{2 k-3}, v_{2 k-2}, v_{2 k}$, and $v_{1}$. Alice colors one of these vertices with color 3 or 4 . Then Bob colors the uncolored vertex at distance 3 with a different color, and one of the remaining uncolored vertices cannot be colored any more. (The colors 3 and 4 are allowed for the vertex Bob has colored since $v_{2 k-5}$ and $v_{3}$ are colored with color 1 .)

In the second case $v_{2 k-5}$ is uncolored and cannot be colored with color 1 . We may assume that $v_{2 k-5}$ can be colored with color 3 . Then Bob colors $v_{2 k-2}$ with color 4 . In order to avoid the situation that $v_{2 k-3}$ cannot be colored any more, Alice must either color $v_{2 k-3}$ with color 3 or $v_{2 k-5}$ with color 4 . Then Bob colors $v_{1}$ with color 3 , and wins since $v_{2 k}$ cannot be colored any more. His move is feasible as we have assumed that $v_{3}$ is colored with color 1 .

The same argumentation does not work for paths with $k \geq 8$ edges. The reason is that in the incidence graph of a cycle, for reasons of symmetry, we can assume without loss of generality that Alice colors $v_{2 k-1}$ in her first move. However, in the incidence graph of a path we cannot assume that Alice starts coloring a vertex in the middle of a path. So we may formulate

Open question. Let $P$ be a large path. Is $\iota_{g}(P)=4$ or $\iota_{g}(P)=5$ ?

\section{Some simple classes of graphs}

Let $S_{k}$ be the star with $k$ edges which are incident with the same center vertex. Further let $W_{k}$ be the wheel with $2 k$ edges. Let $v_{0}$ be the center vertex of a star or a wheel. We call an incidence $\left(v_{0}, e\right)$ inner incidence, and an incidence $(v, e)$ with $v \neq v_{0}$, but where $v_{0}$ is incident with $e$, outer incidence. Incidences on the rim of a wheel, which are neither inner nor outer incidences, are called border incidences. In a star or a wheel, inner incidences are adjacent to all inner and 
outer incidences, but two different outer incidences are not adjacent.

Theorem 4 For $k \geq 1, \iota_{g}\left(S_{2 k}\right)=3 k$.

PROOF. First, we describe a winning strategy for Alice playing on $S_{2 k}$ with $3 k$ colors. At the beginning, Alice colors inner incidences. If Bob colors an outer incidence with color $i$, then Alice also colors an outer incidence with color $i$. In this way, Bob can use at most $k$ colors for outer incidences. If Alice is forced to color an outer incidence (this is only the case when all inner incidences are colored), then she colors an outer incidence with a color already assigned to an outer incidence, except if there is no such color. In the latter case she chooses one of the remaining $k$ colors and continues coloring the outer incidences with that color. Even if Bob always uses a new color for his outer incidences, in his last move he will be forced to take a color already used. In every case, $2 k$ colors are used for inner incidences, and at most $k$ colors for outer incidences, so Alice wins.

Now we discuss a winning strategy for Bob with $3 k-1$ or fewer colors. In his $k$ first moves, Bob colors $k$ outer incidences with $k$ distinct colors. Then there are at most $2 k-1$ colors left to color the inner incidences. Thus it is impossible to color all of them, and Bob wins.

Theorem 5 For $k \geq 0, \iota_{g}\left(S_{2 k+1}\right)=3 k+2$.

PROOF. A winning strategy for Alice playing on $S_{2 k+1}$ with $3 k+2$ colors is given as follows. Alice, in her first $k+1$ moves, colors outer incidences with a fixed color $i$. Meanwhile, Bob can use at most $k$ distinct colors for outer incidences. So $2 k+1$ colors are left for the inner incidences, and Alice wins.

We still have to exhibit a winning strategy for Bob with $3 k+1$ or fewer colors. If Alice, in her first move, colors an inner incidence, Bob, in his first $k+1$ moves, colors outer incidences with $k+1$ distinct colors. On the other hand, if Alice, in her first move, colors an outer incidence with color $i$, then Bob, in his next $k$ moves, colors outer incidences with $k$ distinct colors different from $i$. In both cases, at most $2 k$ colors are left for the inner incidences, which therefore cannot be colored any more, i.e. Bob wins.

Theorem 6 For $k \geq 7, \iota_{g}\left(W_{2 k}\right)=3 k$.

PROOF. First, we describe a winning strategy for Alice with $3 k$ colors which is very similar to the case of stars. Alice chooses 7 colors. During the game, if one of her chosen colors is used for an inner incidence, she exchanges that color with one of the colors not yet used for an inner incidence. This is possible 
since there are $3 k \geq 2 k+7$ colors. As long as Bob colors inner incidences and border incidences, Alice colors inner incidences (or, if she is forced to, border incidences). If Bob colors an outer incidence, Alice replies by coloring an outer incidence with one of her chosen 7 colors, preferably with the same color as Bob (if necessary, Alice exchanges a color not used before among her 7 colors with Bob's color.) There is always a feasible color among these because an outer incidence is affected by at most 4 border incidences. The same strategy will make Alice win if she is forced to color outer incidences first (at the end Bob can also use one of the 7 colors.) Note that border incidences are affected by at most 4 border incidences, at most 2 outer incidences, and one inner incidence. Thus a border incidence can always be colored by one of Alice's 7 colors.

The winning strategy for Bob with $3 k-1$ or fewer colors is just the same as for a star: Bob first colors outer incidences with $k$ distinct colors, then he will win.

Theorem 7 For $k \geq 6, \iota_{g}\left(W_{2 k+1}\right)=3 k+2$.

PROOF. First, we prove a winning strategy for Alice with $3 k+2$ colors. Alice chooses 7 colors. Every time one of these colors is used for inner incidences she exchanges this color with a color not used so far. This is possible since there are $3 k+2 \geq(2 k+1)+7$ colors. In her first move, Alice colors an outer incidence with one of the chosen colors. As long as Bob colors inner or border incidences, Alice colors inner or border incidences as well. If Bob colors an outer incidence, she colors an outer incidence preferably with the same color (and in this case she exchanges this color with one of the seven colors which has not been used before if such a color exists), or else with one of the seven colors, preferably with a color already used. Playing this way Alice can guarantee that the final set of seven colors will be used for outer incidences and that the outer incidences are colored by at most $k+1$ colors. Note that there is no problem coloring the border incidences with these $k+1 \geq 7$ colors. So Alice wins.

A winning strategy for Bob with $3 k+1$ or fewer colors is the same as with stars. In his first $k+1$ moves Bob can guarantee that $k+1$ outer incidences are colored distinctly. Then he will win as the inner incidences cannot be colored any more.

Stars and wheels are classes of graphs for which the incidence game chromatic number is asymptotically half of the value of the trivial upper bound. There is no class of graphs where this fraction is lower because of the following proposition. 
Proposition 8 For any graph $G$ with maximum degree $\Delta, \iota_{g}(G)>\frac{3 \Delta-1}{2}$

PROOF. A winning strategy for Bob with $\left\lceil\frac{3 \Delta}{2}\right\rceil-1$ or fewer colors is given as follows. Bob chooses a vertex $v$ of degree $\Delta$. An outer incidence of $v$ is an incidence $(w, e)$ such that $e=v w$. In his first $\left\lceil\frac{\Delta}{2}\right\rceil$ moves, Bob (possibly with Alice's help) colors $\left\lceil\frac{\Delta}{2}\right\rceil$ outer incidences of $v$ with $\left\lceil\frac{\Delta}{2}\right\rceil$ distinct colors. Then there are only less than $\Delta$ colors left for the incidences of type $(v, e)$. Thus Bob wins.

We have the analogon between the lower and upper bounds for the incidence game chromatic number

$$
\frac{3 \Delta-1}{2}<\iota_{g}\left(G_{\Delta}\right) \leq 3 \Delta-1
$$

and the trivial lower and upper bounds for the game chromatic index

$$
\frac{2 \Delta-1}{2}<\chi_{g}^{\prime}\left(G_{\Delta}\right) \leq 2 \Delta-1
$$

The lower bounds are tight for every $\Delta$ in the sense that no positive integer may be added to them.

Open question. For fixed $\Delta>2$, are the upper bounds in (2) and (3) tight?

Open question. Is the incidence game chromatic number a monotonic parameter (i.e. for a graph $G$ is it as least as big as for any subgraph of $G$ )?

The game chromatic number is known to be non-monotonic (consider $K_{m, m}$ and its subgraph where a perfect matching is deleted). The game chromatic index, too, is non-monotonic (consider $C_{6}$ together with an isolated edge, and its subgraph $P_{6}$ ). Hence, it would be no surprise if the answer of the last question was not affirmative.

\section{Acknowledgements}

Thanks to Artem Pyatkin for pointing out that the proof of Proposition 8 is as easy as it is. Thanks to the anonymous referees for useful suggestions, in particular for the suggestion to improve Theorem 1 to the form in which it is presented here. 


\section{References}

[1] Andres, S. D., The game chromatic index of forests of maximum degree $\Delta \geq 5$, Discrete Appl. Math. 154 (2006), 1317-1323

[2] Bodlaender, H. L., On the complexity of some coloring games, Int. J. Found. Comput. Sci. 2, no.2 (1991), 133-147

[3] Brualdi, R. A., and J. J. Q. Massey, Incidence and strong edge colorings of graphs, Discrete Math. 122 (1993), 51-58

[4] Cai, L., and X. Zhu, Game chromatic index of $k$-degenerate graphs, J. Graph Theory 36 (2001), 144-155

[5] Erdös, P., U. Faigle, W. Hochstättler, and W. Kern, Note on the game chromatic index of trees, Theoretical Comp. Sci. 313 (2004), 371-376

[6] Faigle, U., W. Kern, H. Kierstead, and W. T. Trotter, On the game chromatic number of some classes of graphs, Ars Combin. 35 (1993), 143-150

[7] Guan, D. J., and X. Zhu, Game chromatic number of outerplanar graphs, J. Graph Theory 30, no.1 (1999), 67-70

[8] Guiduli, B., On incidence coloring and star arboricity of graphs, Discrete Math. 163 (1997), 275-278

[9] Hosseini Dolama, M., É. Sopena, and X. Zhu, Incidence coloring of $k$ degenerated graphs, Discrete Math. 283 (2004), 121-128

[10] Huang, C.-I., Y.-L. Wang, and S.-S. Chung, The incidence coloring numbers of meshes, Comput. Math. Appl. 48 (2004), 1643-1649

[11] Kierstead, H. A., A simple competitive graph coloring algorithm, J. Comb. Theory B 78 (2000), 57-68

[12] Liu, X., and Y. Li, The incidence chromatic number of some graph, Int. J. Math. Math. Sci. 2005 (2005), 803-813

[13] Maydanskiy, M., The incidence coloring conjecture for graphs of maximum degree 3, Discrete Math. 292 (2005), 131-141

[14] Wang, S.-D., D.-L. Chen, and S.-C. Pang, The incidence coloring number of Halin graphs and outerplanar graphs, Discrete Math. 256 (2002), 397-405

[15] Zhu, X., Refined activation strategy for the marking game, to appear: J. Comb. Theory B, doi:10.1016/j.jctb.2007.04.004 